\title{
SOLUTIONS OF THE NEUTRAL DIFFERENTIAL-DIFFERENCE EQUATION
}

$$
c\left(x^{\prime}(t)+\beta x^{\prime}(t-r)+\gamma x(t)+\delta x(t-r)=f(t)\right.
$$

\section{LL. G. ChAMBERS}

School of Mathematics University College of North Wales

Dean Street, Bangor, Gwynedd LL57 1UT

United Kingdom

(Received March 29, 1989)

\begin{abstract}
Particular solutions and complementary functions are obtained for the functional equation $\alpha x^{\prime}(t)+\beta x^{\prime}(t-r)+\gamma x(t)+\delta x(t-r)=f(t)$ in the forms of a convolution type integral and of infinite series.
\end{abstract}

KEY WORDS AND PHRASES: Neutral differential-difference equation.

1991 AMS SUBJECT CLASSIFICATION CODES: 35K05.

\section{INTRODUCTION}

Discussions of the neutral differential-difference equation

$$
\alpha x^{\prime}(t)+\beta x^{\prime}(t-r)+\gamma x(t)+\delta x(t-r)=f(t), \quad \alpha, \beta \neq 0
$$

and the corresponding homogeneous equation

$$
\alpha x^{\prime}(t)+\beta x^{\prime}(t-r)+\gamma x(t)+\delta x(t-r)=0
$$

appear to have centered largely about the problem of the solution of the equation when $x(t)$ is given over an interval such as $-r<t<0$, and the behavior of $x$ for $t>0$, and as $t$ tends to infinity [1-5]. It seems that no, or little, attention has been paid to solutions of these equations valid for all $t$. Following the differential equation convention, it will be convenient to refer to any solution of equation (1.1a) as a particular solution, and to regard the solutions of (1.1b) as comprising the complementary function. It may be noted that if $x(t)$ satisfies the homogeneous equation (1.1b), so also does $x(t+\tau)$ where $\tau$ is arbitrary.

It will be shown in the course of this paper that in general three types of solutions to the system of equations (1.1) exist. It may be noted that in all cases relevant convergence criteria must hold for the solution to exist, and no further reference will be made to this.

\section{EXPONENTLAL TYPE SOLUTIONS}

Suppose that an exponential type solution exists to the homogeneous equation (1.1b) of the form $e^{\lambda t}$. The value of $\lambda$ will be given as one of the roots of the characteristic equation

$$
h(\lambda)=0
$$

where

$$
h(\lambda)=(\alpha \lambda+\gamma)+(\beta \lambda+\delta) e^{\lambda r}
$$


This property leads immediately to the possibility of obtaining a particular solution to equation (1.1a) for, if the exciting function on the right hand side of equation (1.1a) is $e^{i \omega t}$, there will be an associated solution $e^{i \omega t} / h(i \omega)$. (In the cases where there are repeated roots to $h(\lambda)=0$, or where $h(i \omega)=0$, there will be terms of the form $t e^{\lambda t}$ or $t e^{i \omega t}$ as appropriate.)

It is now possible to use Fourier Integral Theory [6].

$$
f(t)=\frac{1}{2 \pi} \int_{-\infty}^{\infty} F(\omega) e^{i \omega t} d \omega
$$

where

$$
F(\omega)=\int_{-\infty}^{\infty} f\left(t^{\prime}\right) e^{-i \omega t^{\prime}} d t^{\prime} .
$$

Thus, a particular solution of equation (1.1a) will be given by

$$
x(t)=\frac{1}{2 \pi} \int_{-\infty}^{\infty} \frac{1}{h(i \omega)} F(\omega) e^{i \omega t} d \omega
$$

Let

$$
k(t)=\frac{1}{2 \pi} \int_{-\infty}^{\infty} \frac{1}{h(i \omega)} e^{i \omega t} d \omega .
$$

$k(t)$ may be regarded as a response function, and it follows from the convolution theorem that the relation (2.3a) may be rewritten in the form

$$
x(t)=\int_{-\infty}^{\infty} k\left(t-t^{\prime}\right) f\left(t^{\prime}\right) d t^{\prime}
$$

which gives a particular solution to the equation (1.1a).

\section{A DEGENERATE CASE}

If the relation

$$
\alpha \delta-\beta \gamma=0
$$

be satisfied, it is clearly possible to rewrite equations (1.1a) and (1.1b) (if $\alpha$ be taken as unity which is always possible)

$$
x^{\prime}(t)+\beta x^{\prime}(t-r)+\gamma[x(t)+\beta x(t-r)]-f(t)
$$

Let

$$
z=x(t)+\beta x(t-r)
$$

Then equation (3.1b) takes the form

$$
z^{\prime}(t)+\gamma z(t)=f(t)
$$

the solution of which is

$$
z(t)=C e^{-\gamma t}+e^{-\gamma t} \int_{c}^{t} e^{\gamma t^{\prime}} f\left(t^{\prime}\right) d t^{\prime}
$$

$c$ is arbitrary and can be any convenient value such as zero or positive or negative infinity. Write

$$
z(t)=C e^{-r t}+g(t) \text { say. }
$$

Equation (3.2a) then becomes

$$
x(t)+\beta x(t-r)=C e^{-\gamma t}+g(t)
$$


a particular solution of this corresponding to the first term on the right hand side can easily be seen to be

$$
x(t)=K e^{-r} /\left(1+\beta e^{r}\right)
$$

save that when $1+\beta e^{\gamma r}$ vanishes, the appropriate solution is

$$
x(t)=K t e^{-r t} / r .
$$

It can easily be verified that particular solutions corresponding to

$$
x(t)+\beta x(t-r)=g(t)
$$

are

$$
x(t)=\sum_{s=0}^{\infty}(-\beta)^{s} g(t-s r)
$$

and

$$
x(t)=\sum_{s=0}^{\infty}(-\beta)^{-r} g(t+\overline{s+1} r)
$$

as appropriate.

The homogeneous equation corresponding to equation (3.5a) is

$$
x(t)+\beta x(t-r)=0
$$

which has as solutions $e^{\lambda t}$ where

$$
1+\beta e^{-\lambda r}=0
$$

that is

$$
\lambda=r^{-1}[\log (-\beta)+2 \pi n i]
$$

where $\boldsymbol{n}$ is an arbitrary integer.

If $\beta=1$, the solution is clearly

$$
x(t)=\sum_{s=1}^{\infty}\left(a_{s} \cos \frac{(2 s+1) \pi t}{r}+b_{s} \sin \frac{(2 s-1) \pi t}{r}\right)
$$

and if $\beta=-1$, the solution is given by

$$
x(t)=\sum_{s=0}^{\infty}\left(a_{s} \cos \frac{2 \pi s t}{r}+b_{s} \sin \frac{2 \pi s t}{r}\right) .
$$

It is clear that, even in this degenerate case there is an infinity of functions in the complementary function.

\section{INFINITE SERIES SOLUTIONS IN THE GENERAL CASE}

Before proceeding further, it will be convenient to introduce the notation

$$
I \phi(t)=\int_{c}^{t} \phi\left(t^{\prime}\right) d t^{\prime}
$$

where $c$ is a convenient arbitrary constant. It can easily be shown that

$$
\frac{d}{d t} \int_{e}^{t} \frac{\left(t-t^{\prime}\right)^{n}}{n !} \phi\left(t^{\prime}\right) d t^{\prime}=\int_{e}^{t} \frac{\left(t-t^{\prime}\right)^{n-1}}{(n-1) !} \phi\left(t^{\prime}\right) d t^{\prime}
$$

where $\boldsymbol{n}$ is a positive integer. Thus 


$$
\begin{aligned}
I^{n+1} \phi(t) & -\int_{\epsilon}^{t} \frac{\left(t-t^{\prime}\right)^{n}}{n !} \phi\left(t^{\prime}\right) d t^{\prime} \\
& =\left(\int_{c}^{t}\right)^{n+1} \phi(t) .
\end{aligned}
$$

Two cases will be considered. The first is associated with $f(t)$ vanishing for large negative $t$ and the second for $f(t)$ vanishing for large positive $t$.

In the first case, look for a solution of the form

$$
x(t)=\sum_{n=0}^{\infty} y_{n}(t-n r) .
$$

Substitution of the expression (4.2a) into the equation (1.1a) gives

$$
\alpha \sum_{n=0}^{\infty} y_{n}^{\prime}(t-n r)+\beta \sum_{n=1}^{\infty} y_{n-1}^{\prime}(t-n r)+\gamma \sum_{n=0}^{\infty} y_{n}(t-n r)+\delta \sum_{n=1}^{\infty} y_{n-1}(t-n r)=f(t) \text {. }
$$

Equation (3.2b) is satisfied if

$$
\begin{gathered}
\alpha y_{0}{ }^{\prime}(t)+\gamma y_{0}(t)=f(t) \\
\alpha y_{n}{ }^{\prime}(t)+\gamma y_{n}(t)=-\beta y_{n-1}^{\prime}(t)-\delta y_{n-1}(t) \quad n \geq 1
\end{gathered}
$$

Let

$$
\begin{gathered}
y_{n}(t)=e^{-\gamma t / \alpha} u_{n}(t) \\
y_{n}^{\prime}(t)=e^{-\gamma t / \alpha} u_{n}^{\prime}(t)-\frac{\gamma}{\alpha} e^{-\gamma t / \alpha} u_{n}(t) .
\end{gathered}
$$

Equation (3.3a) becomes

$$
\alpha u_{0}^{\prime}(t)=f(t) e^{-\gamma t / \alpha}=\alpha \psi(t) \text { say }
$$

Equation (3.3b) becomes

$$
\alpha u_{n}^{\prime}(t)=-\beta\left[u_{n-1}^{\prime}(t)-\frac{\gamma}{\alpha} u_{n-1}(t)\right]-\delta u_{n-1}(t)
$$

whence

$$
u_{n}^{\prime}(t)=\lambda u_{n-1}^{\prime}(t)+\mu u_{n-1}(t)
$$

where

$$
\lambda=-\beta / \alpha, \quad \mu=(\beta \gamma-\delta \alpha) / \alpha^{2} .
$$

It will be noted that $\mu=0$ corresponds to the degenerate case discussed in Section 3 .

The solution of equations (4.5a) and (4.5b) for the case of zero $\psi(t)$ will generate the complementary function. As $f(t)$ is small for large negative $t, I \phi(t)$ will have the meaning

$$
\int_{-\infty}^{t} \phi\left(t^{\prime}\right) d t^{\prime}
$$

Thus a formal solution to equations (4.5) is given by 


$$
\begin{aligned}
u_{n}(t) & =(\lambda+\mu J)^{n} I \psi(t) \\
& =\sum_{l=0}^{n}\left(\begin{array}{c}
n \\
l
\end{array}\right) \lambda^{n-l} \mu^{l} I^{l+1} \psi(t) \\
& =\sum_{l=0}^{n}\left(\begin{array}{c}
n \\
l
\end{array}\right) \lambda^{n-1} \mu^{l} \int_{-\infty}^{t} \frac{\left(t-t^{\prime}\right)^{\prime}}{l !} \psi\left(t^{\prime}\right) d t^{\prime} .
\end{aligned}
$$

Thus a particular solution ot the original equation (1.1a) is given by

$$
x(t)=\sum_{n=0}^{\infty} e^{-\gamma(t-n r) \alpha} \sum_{l=0}^{n}\left(\begin{array}{l}
n \\
l
\end{array}\right) \lambda^{n-l} \mu^{l} \int_{-\infty}^{t-n r} \frac{\left(t-n r-t^{\prime}\right)^{l}}{l !} \psi\left(t^{\prime}\right) d t^{\prime} .
$$

It is possible to obtain a bound and a convergence criterion for this infinite series solution.

Suppose that

$$
|\psi(t)|<e^{\mathrm{a} t} \quad \mathrm{a}>0
$$

that is

$$
|f(t)| \alpha^{-1}<e^{(a+\gamma / \alpha) t}
$$

(There is no point in introducing an arbitrary constant of magnitude.) It will also be supposed for convenience that $\lambda$ and $\mu$ are positive and real, and that all the other quantities are real. If this is not the case, only trivial alterations are required.

It can be seen that

$$
\left|\int_{-\infty}^{t-n r} \frac{\left(t-n r-t^{\prime}\right)^{l}}{l !} \psi\left(t^{\prime}\right) d t^{\prime}\right| \leq\left(\int_{-\infty}^{t-n r}\right)^{t+1} e^{\alpha t}=a^{-t-1} e^{\alpha(t-n r)} .
$$

The bound of the summation over $l$ is given therefore by

$$
\mathrm{a}^{-1} e^{2(t-n r)} \sum_{l=0}^{n}\left(\begin{array}{l}
n \\
l
\end{array}\right) \lambda^{n-1} \mu^{l} \mathrm{a}^{-t}=\mathrm{a}^{-1} e^{2(t-n r)}(\lambda+\mu / \mathrm{a})^{n} .
$$

Thus

$$
\begin{aligned}
|x(t)| & \leq \sum_{n=0}^{\infty} e^{-\gamma(t-m r) / \alpha} \mathrm{a}^{-1} e^{\alpha(t-m r)}(\lambda+\mu / \mathrm{a})^{n} \\
& =e^{(\mathrm{a}-\gamma / \mathrm{a}) t} \mathrm{a}^{-1} \sum_{n=0}^{\infty}\left[(\lambda+\mu / \mathrm{a}) e^{\gamma r r / a} e^{-\mathrm{s} r}\right] \\
& =e^{(\mathrm{a}-\gamma / \mathrm{a}) t} \mathrm{a}^{-1}\left(1-\{\lambda+\mu / \mathrm{a}\} e^{\gamma r / a-2 r}\right)^{-1} \\
& =e^{(\mathrm{a}-\gamma / \mathrm{a}) t}\left(\mathrm{a}-\{\lambda \mathrm{a}+\mu\} e^{\gamma / \alpha-a r}\right)^{-1} .
\end{aligned}
$$

The summation is valid if

$$
\left|(\lambda+\mu / a) e^{-(a-\gamma / a) r}\right|<1 .
$$

The complementary function set may be obtained by the interpretation

$$
I^{l+1} 0=\sum_{k=0}^{l} c_{k} \frac{t^{l-k}}{(l-k) !}
$$

where the $c_{k}$ are arbitrary constants. This generates a complementary function system for the set (4.5) 


$$
\begin{aligned}
u_{n}(t) & =\sum_{l=0}^{n}\left(\begin{array}{l}
n \\
l
\end{array}\right) \lambda^{n-l} \mu^{l} \sum_{k=0}^{l} c_{k} \frac{t^{l-k}}{(l-k) !} \\
& =\sum_{k=0}^{n} c_{k} \sum_{l=k}^{n}\left(\begin{array}{l}
n \\
l
\end{array}\right) \lambda^{n-l} \mu^{\prime} \frac{t^{l-k}}{(l-k) !} .
\end{aligned}
$$

The associated complementary function set for the original system is therefore

$$
\begin{aligned}
& \sum_{n=0}^{\infty} e^{-\gamma(t-n r) \alpha} \sum_{k=0}^{n} c_{k} \sum_{t=k}^{n}\left(\begin{array}{l}
n \\
l
\end{array}\right) \lambda^{n-l} \mu^{l} \frac{(t-n r)^{t-k}}{(l-k) !} \\
& =\sum_{k=0}^{\infty} c_{k} \sum_{n=k}^{\infty} e^{-\gamma(t-n r) \alpha} \sum_{l=k}^{n} \lambda^{n-l} \mu^{l} \frac{(t-n r)^{t-k}}{(l-k) !} \\
& -\sum_{k=0}^{\infty} c_{k} Y_{k}(t)
\end{aligned}
$$

where

$$
Y_{k}(t)=\sum_{n=k}^{\infty} e^{-\gamma(t-n r) / \alpha} \sum_{l=k}^{n}\left(\begin{array}{l}
n \\
l
\end{array}\right) \lambda^{n-l} \mu^{l} \frac{(t-n r)^{\gamma-k}}{(l-k) !} .
$$

As the $c_{k}$ are arbitrary, the $Y_{k}(t)$ form an infinite set comprising the complementary function.

Suppose now that $f(t)$ vanishes for large positive $t$. Look for a solution of the form

$$
x(t)=\sum_{n=0}^{\infty} Z_{n}(t+n r) .
$$

Then

$$
\begin{aligned}
\alpha \sum_{n=0}^{\infty} Z_{n}^{\prime}(t+n r)+\beta \sum_{n-1}^{\infty} Z^{\prime}{ }_{n+1}(t+n r)+\gamma \sum_{n=0}^{\infty} Z_{n}(t+n r) \\
+\delta \sum_{n=-1}^{\infty} Z_{n+1}(t+n r)=f(t+r-r) .
\end{aligned}
$$

Equation (4.12b) is satisfied if

$$
\begin{gathered}
\beta Z_{0}^{\prime}(t)+\delta Z_{0}(t)=f(t-r) \\
\beta Z_{n+1}^{\prime}(t)+\delta Z_{n+1}(t)=-\alpha Z_{n}(t)-\gamma Z_{n}(t) \quad n \geq 0 .
\end{gathered}
$$

Let

$$
\begin{gathered}
Z_{n}(t)=e^{-\delta / / \beta} V_{n}(t) \\
Z_{n}^{\prime}(t)=e^{-\Delta t / \beta} V_{n}^{\prime}(t)-\frac{\delta}{\beta} e^{-\delta t / \beta} V_{n}(t)
\end{gathered}
$$

Equation (4.13a) becomes

$$
\beta V_{n}^{\prime}(t)=f(t-r) e^{-s t / \beta}=\beta \theta(t) \text { say. }
$$

Equation (4.13b) becomes

$$
\beta V_{n}^{\prime}(t)=-\alpha\left[V_{n-1}^{\prime}(t)-\frac{\delta}{\beta} V_{n-1}(t)\right]-\gamma \delta V_{n-1}(t)
$$

whence

$$
V_{n}^{\prime}(t)=\xi V_{n-1}^{\prime}(t)+\eta V_{n-1}(t)
$$

where

$$
\xi=-\alpha / \beta, \quad \eta=(\alpha \delta-\beta \gamma) / \beta^{2} .
$$


It will be noted that $\boldsymbol{\eta}=\mathbf{0}$ corresponds to the degenerate case.

The solution of equations (4.15a) and (4.15b) for the case of zero $\theta(t)$ will generate the complementary function. As $f(t)$ is small for large positive $t, I \phi(t)$ will have the meaning

$$
-\int_{1}^{\infty} \phi\left(t^{\prime}\right) d t^{\prime} \text {. }
$$

The process proceeds exactly as previously, and will give rise to a solution

$$
x(t)=\sum_{n=0}^{\infty} e^{-\alpha(t+n r) \beta} \sum_{t=0}^{n}\left(\begin{array}{l}
n \\
l
\end{array}\right) \xi^{n-l} \eta^{\prime}\left[-\int_{t+n r}^{\infty} \frac{\left(t+n r-t^{\prime}\right)^{\prime}}{l !} \theta\left(t^{\prime}\right) d t^{\prime}\right]
$$

A convergence criterion and bound for $x(t)$ may be determined in exactly the same way as previously. If

$$
|\theta(t)|<e^{-b x} \quad b>0
$$

that is

$$
|f(t-r)| \beta^{-1}<e^{-(b-\delta \beta) t}
$$

or

$$
|f(t)| \beta^{-1}<e^{-(b-\alpha \beta)(t+r)}
$$

the bound for the integral is

$$
b^{-(l+1)} e^{-b(t+n r)}
$$

and the bound for the summation over $l$ is given therefore by

$$
b^{-1} e^{-b(t+n r)} \sum_{l=0}^{n}\left(\begin{array}{l}
n \\
l
\end{array}\right) \xi^{n-l} \mu^{l} b^{-t}=b^{-1} e^{-b(t+n r)}(\xi+\eta / b)^{n} .
$$

Thus

$$
\begin{aligned}
&|x(t)| \leq \sum_{n=0}^{\infty} e^{-\delta(t+a r) \beta \beta} b^{-1} e^{-b(t+n r)}(\xi+\eta / b)^{n} \\
&=e^{-(b+\alpha \beta \beta) t} b^{-1} \sum_{n=0}^{\infty}(\xi+\eta / b) e^{-(b+\alpha \beta) r} \\
&=e^{-(b+\alpha \beta)}\left(b+\{\xi b+\eta\} e^{-(b+\alpha \beta) r}\right)^{-1} .
\end{aligned}
$$

The summation is valid if

$$
\left|(\xi+\eta / b) e^{-(b+\Delta \beta) r}\right|<1 .
$$

In the same way as previously, a complementary function set

$$
Z_{k}(t)=\sum_{n=k}^{\infty} e^{-\alpha(t+n r) \beta} \sum_{l=k}^{n}\left(\begin{array}{l}
n \\
l
\end{array}\right) \xi^{n-l} \eta^{l} \frac{(t+n r)^{l-k}}{(l-k) !}
$$

may be found.

\section{DISCUSSION}

It may be noted that if $\beta$ is zero, the discussion involving equations (4.1) to (4.11) remains valid and that if $\alpha$ is zero the discussion involving equations (4.12) to (4.18) remains valid. In these cases however, the equation is no longer neutral.

If instead of equation (1.1a), the equation is

$$
A x^{\prime}(t)+B x^{\prime}(t-r)+C x(t)+D x(t-r)=f(t)
$$


where $A, B, C, D$, are square matrices and $x$ is a column vector, the whole of the previous analysis may be carried out provided that the following conditions hold

a) where previously $\alpha$ and $\beta$ respectively are non zero $A^{-1}$ and $B^{-1}$ exist.

b) $e^{p}$ is interpreted as

$$
\sum_{s=0}^{\infty} \frac{p^{s}}{s}, P^{0} \text { being the unit matrix. }
$$

c) $\alpha^{-1} \gamma$ is replaced by $A^{-1} C, \beta^{-1} \delta$ by $B^{-1} D$. $\alpha / \beta$ by $B^{-1} A, \beta / \alpha$ by $A^{-1} B,(\alpha \delta-\beta \gamma) / \beta^{2}$ by $B^{-2}(A D-B C)$ and $(\beta \gamma-\delta \alpha) / \alpha^{2}$ by $A^{-2}(B C-A D)$.

d) When convergence is considered norms will be involved, rather than moduli

$$
e g\|\psi(t)\|<e^{\alpha} .
$$

\section{REFERENCES}

[1] BRAYTON, R. K. Q. App. Math. 24, 1966, 215.

[2] HALE, J. K. and MEYER, K. R. A class of functional equations of neutral type, Mem. Amer. Math. Soc., No. 76 (American Mathematical Society, Providence, Rhode Island), 1967, 32.

[3] HALE, J. Theory of Functional Differential Equations (Springer-Verlag, Berlin), 1977, 24.

[4] BELLMAN, R. and COOKE, K. L. Differential-Difference Equations (Academic Press, New York), $1963,143$.

[5] HENRY, D. J. Diff. Eq. 15, 1974, 106.

[6] PAGE, C. H. Physical Mathematics (D van Nostrand Company, Inc., Princeton, New Jersey), 1955, 192. 


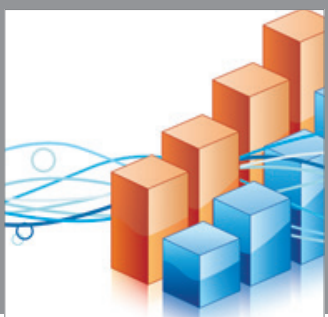

Advances in

Operations Research

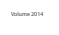

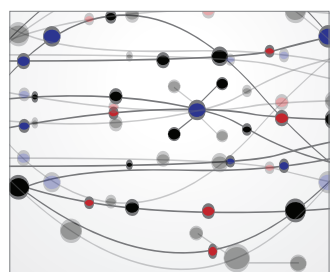

\section{The Scientific} World Journal
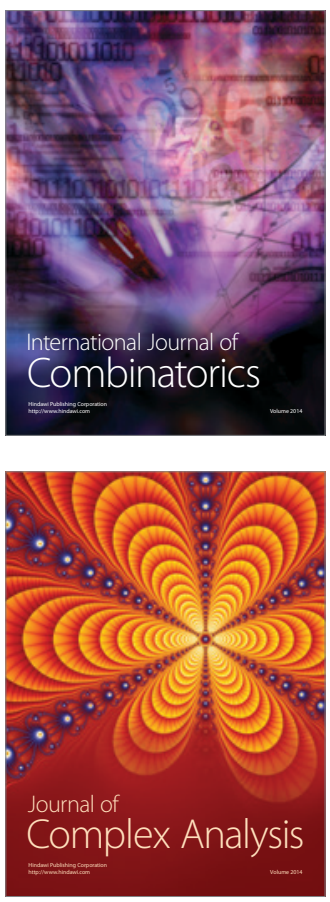

International Journal of

Mathematics and

Mathematical

Sciences
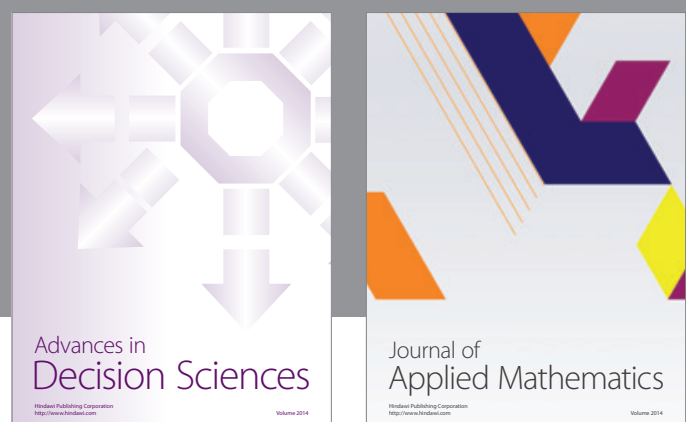

Journal of

Applied Mathematics
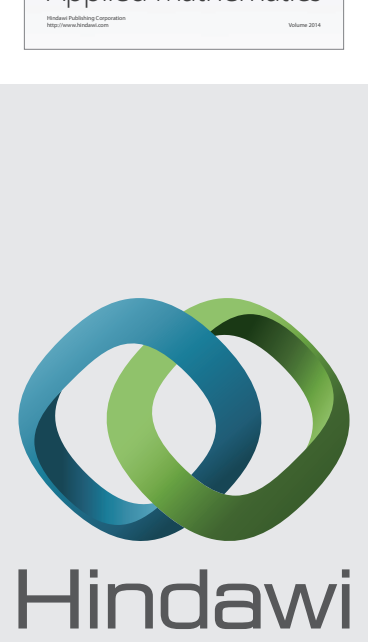

Submit your manuscripts at http://www.hindawi.com
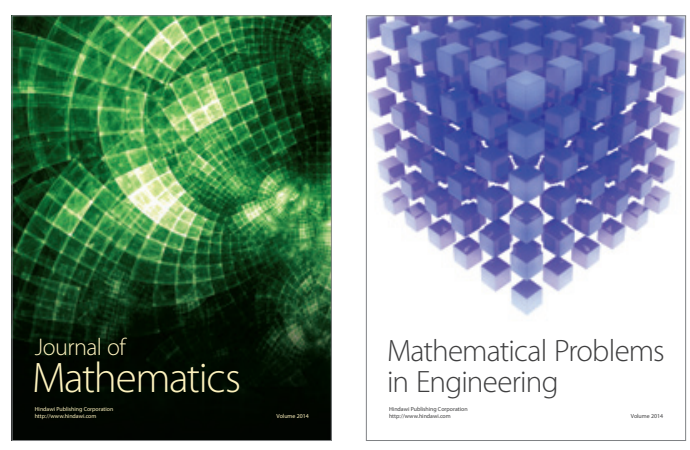

Mathematical Problems in Engineering
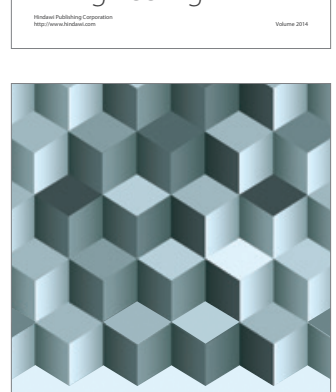

Journal of

Function Spaces
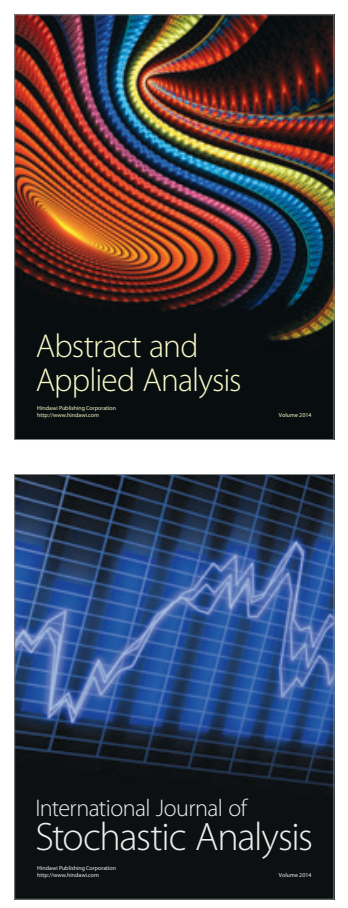

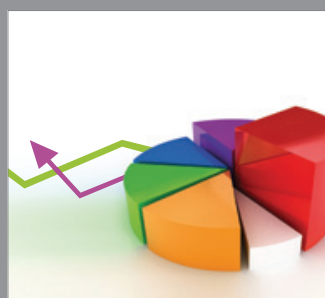

ournal of

Probability and Statistics

Promensencen
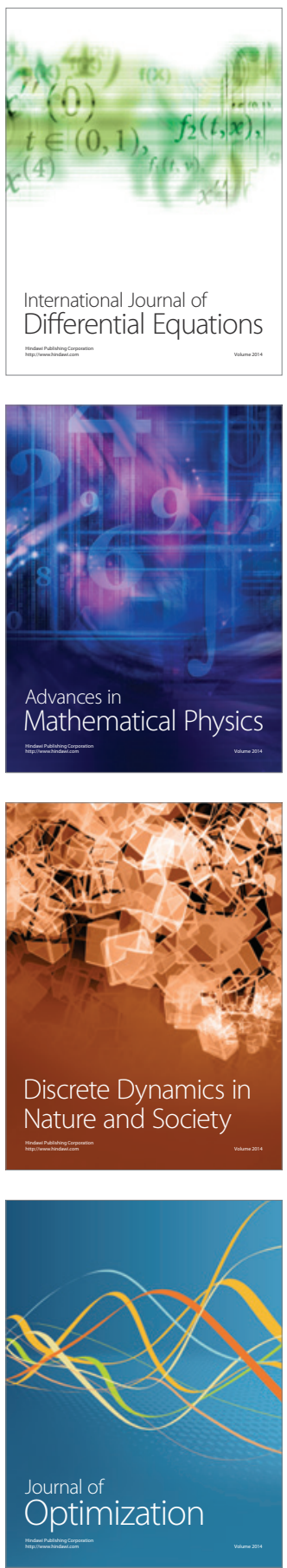